\title{
Epilepsy caused by BRAF-mutated paediatric brain tumours gets a REST
}

Paediatric brain tumours often cause epileptic seizures that are resistant to therapies. A new article in Nature Medicine shows that BRAF-V600E - an oncogenic mutation that is often found in melanomas - is also found in about half of epilepsyassociated paediatric brain tumours. In a mouse model of these tumours, vemurafenib, an approved drug for BRAF-mutant metastatic melanoma, alleviated seizures.

Ganglioglioma is a low-grade tumour that is strongly associated with epilepsy. The authors found that in 56 low-grade gliomas, half of the tumours had mutations in $B R A F$, and in the subset of patients with ganglioglioma the percentage rose to $60 \%$. Introduction of BRAF-V637E (the orthologue of the BRAF-V600E mutation) into a subset of neurons and glia in mice induced dysmorphic, but not neoplastic, neurons and glial cells with features reminiscent of ganglioglioma. Importantly, $>90 \%$ of these mice had seizures at postnatal week 4 . Seizures were associated with increased rates of spontaneous firing and increased synchronicity of the burst firings.

RNA sequencing of BRAFV600E tumours from patients with ganglioglioma showed that genes involved in synaptic transmission, nervous system development and regulation of synaptic plasticity (particularly ion channels and neurotransmitter receptors) were downregulated, and those genes involved in antigen processing and presentation were upregulated. Many of the downregulated ion channels and receptors were found to be potentially controlled by

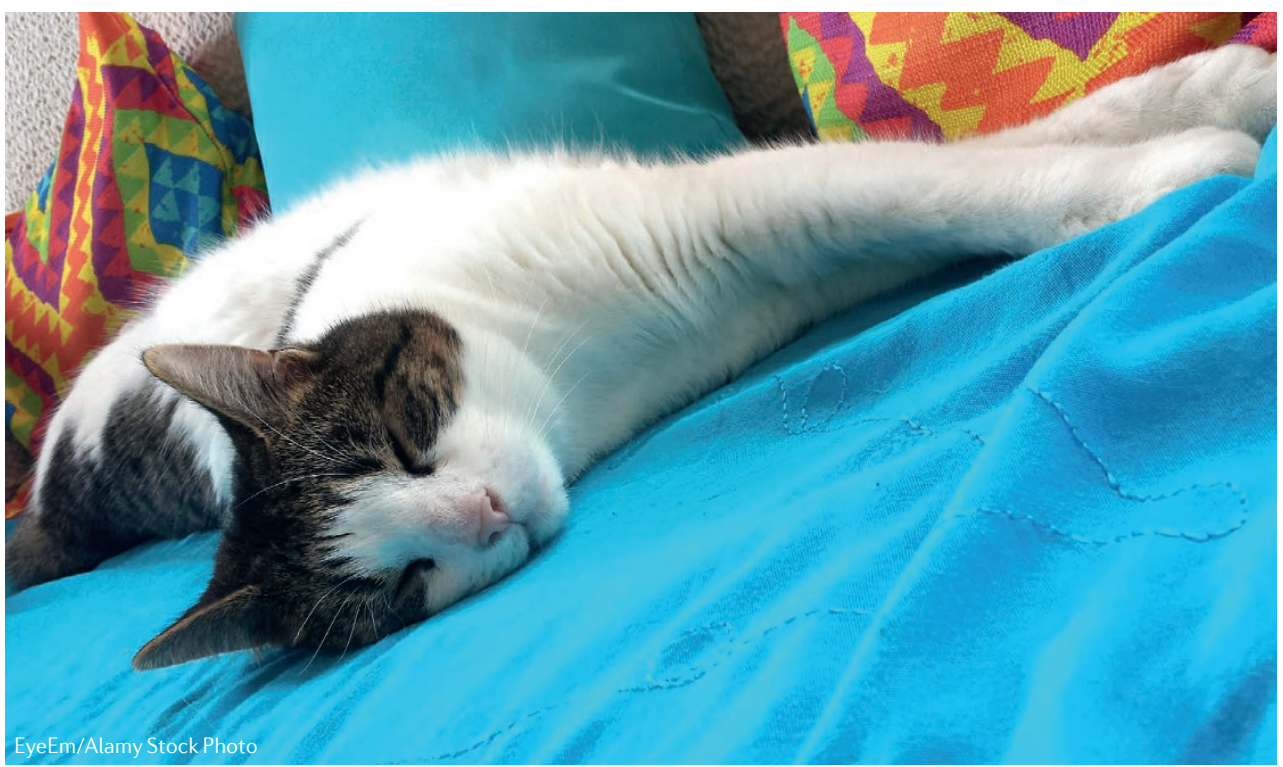

RE1-silencing transcription factor (REST) using upstream regulator analysis. Indeed, REST expression was elevated in neuronal cells of patients with BRAF-V600E tumours and in the mouse models of those tumours. Further analysis of upregulated transcription factors suggested that MYC activation, downstream of BRAF activity, could serve as the link between BRAF-V600E and REST upregulation.

The authors then investigated whether inhibiting BRAF-V600E, using the FDA-approved drug vemurafenib, could alleviate seizures in mouse models. Although oral administration was ineffective, intracerebroventricular infusion of vemurafenib using an osmotic pump reduced the frequency and severity of seizures. Similarly, electroporation of dominant-negative forms of REST into embryos with activated BRAF delayed the onset of seizures and reduced their frequency and severity.

These results demonstrate that BRAF-V600E is a common mutation in intractable paediatric brain tumours. The authors highlight that intracerebroventricular administration is not practical in the clinical setting. However, a brain-penetrant oral BRAF inhibitor could potentially be developed and used to treat paediatric patients with BRAFV600E-positive, epilepsy-associated gliomas and ganliogliomas.

Megan Cully

ORIGINAL ARTICLE Koh, H. Y. et al.

BRAF somatic mutation contributes to intrinsic epileptogenicity in pediatric brain tumors. Nat. Med. https://doi.org/10.1038/s41591018-0172-x (2018) 